[RAdiocarbon, Vol. 20, No. 2, 1978, P. 234-244]

\title{
PHYSICAL RESEARCH LABORATORY \\ RADIOCARBON DATE LIST III
}

\author{
D P AGRAWAL, R V KRISHNAMURTHY, \\ SHEELA KUSUMGAR, and R K PANT
}

Physical Research Laboratory, Ahmedabad-380009, India

Presented below are dates from some important archaeologic and Quaternary sites. All dates are based on $\tau 1 / 2=5568 \mathrm{yr}$; to convert the radiocarbon dates for archaeologic samples into $\mathrm{AD} / \mathrm{BC}$ scale, 1950 has been used as base as per the resolution passed at the Ninth International Radiocarbon Conference, San Diego, 1976. The dates are not corrected for ${ }^{13} \mathrm{C}$ fractionation. All the dates older than 10,000 yr have been given with $2 \sigma$ errors. Due to uncertainty about the contribution of the biogenic carbon in caliche $\left(\mathrm{CaCO}_{3}\right)$ samples, the dates represent apparent and not true ages.

Samples were converted to methane for measuring ${ }^{14} \mathrm{C}$ activity in gas proportional counters. Detailed techniques were described earlier (R, 1971, v 13, p 442-449). All archaeologic samples were given $\mathrm{NaOH}$ pretreatment.

General Comment: many dates on Pacific Ocean sediments (PRL-284286, PRL-326-329, PRL-332, PRL-344-346, PRL-348-357, and PRL-360372) were obtained to determine sedimentation rates. ${ }^{14} \mathrm{C}$ dates on caliche formations of Rajasthan were measured to date climatic changes depicted by sedimentary profiles. Wherever possible measurements were based on $\mathrm{CO}_{2}$ evolved from outer (a), middle (b), and core (c) fractions of the same nodule which enabled a study of the growth rates of caliche nodules. The ${ }^{14} \mathrm{C}$ dated pollen sequence from Toshmaidan (PRL-2B-5, PRL-7, PRL-9-10 and PRL-12) indicates that deglaciation in Kashmir valley started ca 15,000 yr ago. The Ramtirth Ware, a newly discovered Deccan Chalcolithic culture, has been dated to 3500 yr BP (PRL-382-384).

\section{ARCHAEOLOGIC SAMPLES}

\section{Aligrama series, Pakistan}

Aligrama $\left(34^{\circ} 49^{\prime} \mathrm{N}, 72^{\circ} 19^{\prime} \mathrm{E}\right)$, Dist Swat, Pakistan; subm by Sebastiano Tusa, ISMEO, Rome, Italy. Comment: samples were measured to date Swat culture deposits (Stacul \& Tusa, 1975, p 291-321).

PRL-243. Swat culture Vth period

$2900 \pm 110$

Charcoal, Loc Tr E, Layer 9, depth 4.5m.

PRL-244. Swat culture Vth period

$2660 \pm 130$

Charcoal, Loc Tr F, Area 3, Layer 4, depth 2.5m.

PRL-246. Swat culture Vth period

$3080 \pm 170$

Charcoal, Loc Tr F, Area 4, Layer 7, depth 5.5m. 


\section{Apegaon series, Maharashtra}

Apegaon, a Chalcolithic site, Dist Aurangabad; subm by S B Deo, Deccan Coll, Poona. Comment: site has yielded a new ceramic viz Ramtirth Ware, different from the other Chalcolithic (Jorwe and Malwa) wares of the Deccan.

\section{PRL-382. Chalcolithic deposit}

$$
3450 \pm 100
$$

Charcoal, Loc Tr XII-XVI, Layer 4, depth 1.3m.

PRL-383. Chalcolithic deposit

$$
3450 \pm 100
$$

Charcoal, Loc Tr XII-XVI, Layer 5, depth 1.5m.

PRL-384. Chalcolithic deposit

$$
3520 \pm 100
$$

Charcoal, Loc Tr XII-XVI, Layer 6.

\section{PRL-283. Besnagar, India, Northern Black}

$$
\text { Polished (NBP) Ware deposit }
$$

$$
2200 \pm 130
$$

Charcoal from Besnagar $\left(23^{\circ} 30^{\prime} \mathrm{N}, 77^{\circ} 45^{\prime} \mathrm{E}\right)$, Dist Vidisha, Loc Tr BSN-VI, Layer 11, depth 4.1m; subm by Dir Gen Archaeol, New Delhi.

\section{Bhalukpung series, Arunachal Pradesh}

Bhalukpung $\left(27^{\circ} 30^{\prime} \mathrm{N}, 92^{\circ} 20^{\prime} \mathrm{E}\right)$, Dist Kameng, subm by $\mathrm{B} \mathrm{M}$ Das, Dibrugarh Univ, Assam.

PRL-287. Terrace deposit

Carbonized rice, $\operatorname{Tr} 1$, Loc Dezenling, Layer 2, depth .3 to $1 \mathrm{~m}$; sender's Sample S/B/1.

PRL-288. Terrace deposit $650 \pm 80$

Charcoal, Tr 1, Loc Dezenling, Layer 2, depth .3 to 1m; sender's Sample S/B/2.

\section{Bhimbetka series, Madhya Pradesh}

Bhimbetka $\left(22^{\circ} 65^{\prime} \mathrm{N}, 77^{\circ} 57^{\prime} \mathrm{E}\right)$, Dist Raisen, subm by V N Misra, Deccan Coll, Poona. Comment: dates show scatter indicating probably admixture due to recent fire-building activity at site.

PRL-306. Cave deposit

Charcoal, Tr F, Loc 1 \& 2, Layer 1, depth .46 to .5m; sender's Sample BTK-IIIF-13-1976-1.

PRL-310. Cave deposit

Charcoal, Tr F, Loc 1 \& 2, Layer 2, depth .5 to .55m; sender's Sample BTK-IIIF-13-1976-2.

\section{PRL-311. Cave deposit}

Charcoal, Tr F, Loc 1 \& 2, Layer 2, depth .56 to .6m; sender's Sample IIIF-13-1976-3. 
PRL-314. Cave deposit, burial

Charcoal, Tr E, Loc 2, Layer 2, depth .61 to .65m; sender's Sample BTK-IIIF-13-1976-8.

PRL-315. Cave deposit

$1760 \pm 180$

Charcoal, Tr F, Loc 1 \& 2, Layer 2, depth .61 to .65m; sender's Sample BTK-IIIF-13-1976-4.

PRL-316. Cave deposit

$1930 \pm 100$

Charcoal, Tr F, Loc 2, Layer 2, depth .66 to .7m; sender's Sample BTK-IIIF-13-1976-5.

PRL-317. Cave deposit

$2490 \pm 100$

Charcoal, Tr E, Loc 2, Layer 2, depth .66 to .7m; sender's Sample BTK-IIIF-13-1976-9.

PRL-318. Cave deposit

$3560 \pm 100$

Charcoal, Tr E, Loc 1, Layer 3, depth .71 to .75m; sender's Sample BTK-IIIF-13-1976-7.

PRL-321. Cave deposit

$\mathbf{3 7 0} \pm \mathbf{1 3 0}$

Charcoal, Tr E, Loc 2, Layer 3, depth 1.06 to 1.1m; sender's Sample BTR-IIIF-13-1976-11.

\section{PRL-325. Ganwaria, India, Painted Grey Ware (PGW)}

$$
\text { deposit } 4610 \pm 110
$$

Ganwaria $\left(27^{\circ} 26^{\prime} \mathrm{N}, 83^{\circ} 7^{\prime} \mathrm{E}\right)$, Dist Basti, Loc Tr XAl Qd2, Rm 17, Layer 15, depth 7m; subm by Dir Gen Archaeol, New Delhi, sender's Sample 15. Comment: date represents old charcoal and has no relevance to cultural levels.

PRL-253. Hatti, India, Old gold mining $2630 \pm 150$

Charred wood from ancient shaft at Hatti, Dist Raichur, subm by Dir Gen Archaeol, New Delhi; sender's Sample 2/75/MSC.

PRL-252. Ingaladhal, India, Old copper working $1680 \pm 100$

Wood from ancient shaft at Ingaladhal, Dist Chitradurga, subm by Dir Gen Archaeol, New Delhi; sender's Sample 1/75/MSC.

\section{Jodhpura series, India}

Jodhpura $\left(27^{\circ} 31^{\prime} \mathrm{N}, 76^{\circ} 5^{\prime} \mathrm{E}\right)$ Dist Jaipur, subm by Dir Archaeol \& Mus, Jaipur.

PRL-272. Painted Grey Ware $(P G W)$ deposit $2670 \pm 150$ $3 / 75$.

Charcoal, Loc Tr D, Layer 12, depth 1.97m; sender's Sample JRA

PRL-273. PGW deposit

$2310 \pm 140$

Charcoal, Loc Tr E, Layer 8, depth 2.5m; sender's Sample JRA 4/75. 
PRL-274. PGW deposit

$2250 \pm 110$

Charcoal, Loc Tr D, Layer 12, depth 2.9m; sender's Sample JRA $5 / 75$.

PRL-275. Black-and-Red Ware (BRW)

$$
\text { deposit (?) }
$$
$6 / 75$.

Charcoal, Loc Tr D, Layer 13, depth 2.87m; sender's Sample JRA

PRL-277. Ocher Color Pottery (OCP)

$$
\text { deposit (?) } 2610 \pm 110
$$
$9 / 75$.

Charcoal, Loc Tr D, Layer 14, depth 1.94m; sender's Sample JRA

PRL-278. OCP deposit

$$
4060 \pm 170
$$

Charcoal, Loc Tr D, Layer 14, depth 3m; sender's Sample 12/75.

PRL-254. Kalyadi, India, Old copper working $\quad 310 \pm 80$

Wood from an ancient shaft at Kalyadi, Dist Hassan, subm by Dir Gen Archaeol, New Delhi; sender's Sample 3/75/MSC.

\section{Mitathal series, Haryana}

Mitathal $\left(28^{\circ} 50^{\prime} \mathrm{N}, 76^{\circ} 10^{\prime} \mathrm{E}\right)$, Dist Bhiwani, subm by Suraj Bhan, Ind Inst Adv Studies, Simla.

PRL-290. Late Siswal culture (?) $\quad 3820 \pm 130$ 10.

Charcoal, Loc Tr MTL-1, Layer 17, depth 2.95m; sender's Sample

PRL-291. Harappa culture

$3600 \pm 110$

Charcoal, Loc Tr MTL-1, pit sealed by Layer 10, depth 2.6m; sender's Sample 11.

PRL-292. Harappa culture

$4210 \pm 210$

Charcoal, Loc Tr MTL-2, pit sealed by Layer 4; sender's Sample 15.

\section{Mathura series, Uttar Pradesh}

Mathura $\left(27^{\circ} 28^{\prime} \mathrm{N}, 77^{\circ} 42^{\prime} \mathrm{E}\right)$, Dist Mathura, subm by Dir Gen Archaeol, New Delhi.

PRL-333. Northern Black Polished Ware (NBP)

$$
\text { deposit } 2490 \pm 140
$$

Charcoal, Tr MTR-8, Loc B1 Qd2, Pit 3 sealed by Layer 5, depth $3.18 \mathrm{~m}$; sender's Sample 1.

PRL-334. NBP deposit

Charcoal, Tr MTR-10, Loc A1 Qd4, Layer 9, depth 1.45m; sender's Sample 3. 
PRL-336. NBP deposit

$$
2540 \pm 90
$$
Sample 5 .

Charcoal, Tr MTR-8, Loc B1 Qd2, Layer 6, depth 3m; sender's

PRL-337. NBP deposit

Charcoal, Tr MTR-11, Loc Trial Trench (TT), Pit 5 sealed by Layer 20, depth 5.45m; sender's Sample 6.

PRL-338. NBP deposit $2280 \pm 100$ Sample 7.

Charcoal, Tr MTR-11, Loc TT, Layer 18, depth 4.7m; sender's

PRL-339. NBP deposit

$2380 \pm 100$

Charcoal, Tr MTR-8, Loc B1 Qd3, Pit 2 sealed by Layer 3; sender's Sample 8.

PRL-340. PGW-NBP overlap (?)

$2390 \pm 150$

Charcoal, Tr MTR-8, Loc Al Qd4, Layer 11, depth 4.2m; sender's Sample 9.

PRL-342. PGW-NBP overlap (?)

$2180 \pm 160$

Charcoal, Tr MTR-8, Loc B1 Qd2, Pit 8 sealed by Layer 8, depth 3.6m; sender's Sample 11.

PRL-343. NBP deposit $2150 \pm 100$

Charcoal, Tr MTR-11, Loc TT, Layer 18, depth 4.2m; sender's Sample 12.

\section{Piprahwa series, Uttar Pradesh}

Piprahwa $\left(27^{\circ} 26^{\prime} \mathrm{N}, 83^{\circ} 7^{\prime} \mathrm{E}\right)$, Dist Basti, subm by Dir Gen Archaeol, New Delhi.

PRL-322. Sunga-Kushana deposit

$2250 \pm 100$

Charred rice, NW corner room of E Monastery, Layer 2, depth $1.65 \mathrm{~m}$; sender's Sample 10.

PRL-323. Pre-Mauryan deposit Sample 13.

Charcoal, Room 2, E Monastery, Layer 8, depth 4.1m; sender's

PRL-324. Pre-Mauryan deposit

$2170 \pm 130$

Charcoal, Tr ZA2 Qd3, Layer 8, depth 4.2m; sender's Sample 14.

\section{Pirak series, Pakistan}

Pirak $\left(29^{\circ} 30^{\prime} \mathrm{N}, 67^{\circ} 54^{\prime} \mathrm{E}\right)$ a Chalcolithic site, Dist Kachi, subm by $\mathrm{J}$ F Jarrige, Mus Guimet, Paris.

PRL-388. Chalcolithic deposit $2730 \pm 110$

Charcoal, Tr PKG 3G, Loc CXIII, Layer 2, depth 1.1m. 
PRL-389. Chalcolithic deposit

$2590 \pm 100$

Charcoal, Tr PKC 2F, Loc CVII, Layer 7, depth 1.6m.

PRL-390. Chalcolithic deposit

$2730 \pm 100$

Charcoal, PKA 3G, Loc LXXVII, Layer 21W, depth 3m.

PRL-391. Chalcolithic deposit

$2730 \pm 100$

Charcoal, Tr PKA 3I, Layer 42N, depth 10m.

PRL-298. Zawar, India, lead workings

Modern

Carbon from lead smelting retort coll at Zawar $\left(24^{\circ} 21^{\prime} \mathrm{N}, 73^{\circ} 41^{\prime}\right.$

E), Dist Udaipur; subm by G P Deshmukh, Geol Survey, Jaipur.

II. QUATERNARY SAMPLES

\section{PRL-385. Admiralty Bay, Antarctica,} morainic deposit

Wood from a crag slope at Admiralty Bay $\left(62^{\circ} \mathrm{S}, 64^{\circ} \mathrm{W}\right)$ Antarctica, subm by E Anati, Centro Camuno Studi Prehist, Capo Di Ponte, Italy.

PRL-146. Andada, India, river terrace deposit

$31,830+2660$

Lime-caliche from Upper Terrace on Narmada $\mathrm{R}$ near Andada, Broach Dist, depth 3m; subm by N Bedi, Geol Survey India, Ahmedabad; sender's Sample GSI/NB/3.

PRL-42. Bombay High, India, continental shelf $\quad 11,120_{-300}^{+320}$

Shelf sediment from $0.4 \mathrm{~m}$-long drill core off Bombay. Subm by B $\mathrm{S}$ Venkatachala, Palynol Lab, Dehra Dun. Sender's Sample V2/H-1-1/P.

\section{Chirai series, Rajasthan}

Chirai, Jodhpur Dist; subm by D P Agrawal, PRL, Ahmedabad. Comment: samples measured to study caliche formation.

\begin{tabular}{lr} 
PRL-377. Caliche & $\mathbf{2 7 , 8 2 0}+\mathbf{2 6 9 0}$ \\
\hline & $\mathbf{2 0 1 0}$
\end{tabular} C-22.

Caliche from exposed sec at Chirai, depth 1.65m; sender's Sample

PRL-378. Caliche

$21,550+600$ C-23.

Caliche from exposed sec at Chirai, depth $1.3 \mathrm{~m}$; sender's Sample

\section{PRL-379. Caliche}

$22,350+640$ C-24.

Caliche from exposed sec at Chirai, depth 1.15m, sender's Sample

PRL-262. Chotila, India, miliolite deposit

$15,820+690$

Miliolite from hillslope near Chotila, Surendranagar Dist, depth $0.1 \mathrm{~m}$; subm by B Roy, PRL, Ahmedabad. Comment: sample measured to date inland miliolite. 


\section{PRL-191. Dungarpur, India, miliolite deposit}

$19,780+850$

Miliolite from Dungarpur, Junagarh Dist, depth $12.3 \mathrm{~m}$; subm by D P Agrawal, PRL, Ahmedabad. Comment: sample measured to date miliolite formation.

\section{Gudlai Nadi series, Rajasthan}

Gudlai Nadi, Jodhpur Dist; subm by D P Agrawal, PRL, Ahmedabad. Comment: samples measured to study caliche formation.

PRL-373. Caliche

Caliche from exposed sec of Gudlai Nadi R; horizon D, depth $0.5 \mathrm{~m}$; sender's Sample C-20.

a) $14,960+210$

PRL-374. Caliche

b) $22,910+930$

c) $18,310+\mathbf{5 8 0}$

Caliche from exposed sec on Gudlai Nadi R from horizon C, depth $1.4 \mathrm{~m}$; sender's Sample C-19.

\section{PRL-375. Caliche}

Caliche from exposed sec on Gudlai Nadi R; depth $1.8 \mathrm{~m}$; sender's Sample C-18.

PRL-376. Caliche

Caliche from exposed sec on Gudlai Nadi R; depth $3.2 \mathrm{~m}$; sender's Sample C-17.

PRL-263. Junagarh, India, miliolite deposit

$\mathbf{3 3 , 7 5 0}+\mathbf{3 2 5 0}$

Miliolite from dune at base of Girnar Hill, Junagarh Dist; subm by B Roy, PRL, Ahmedabad.

PRL-236. Kolara, India, terrace deposit

Peaty clay with wood fragments from river terrace near Kolara $\left(22^{\circ} 30^{\prime} \mathrm{N}, 88^{\circ} 30^{\prime} \mathrm{E}\right)$, Howrah Dist; depth $6.65 \mathrm{~m}$; subm by H P Gupta, Birbal Sahni Inst Palaebot, Lucknow. Comment: dated to study possible submergence of forest in Bengal Basin.

\section{Little Rann of Kutch series, Gujarat}

Little Rann of Kutch, Surendranagar Dist; subm by R S Kathiara, Geol Survey, Ahmedabad. Comment: samples measured to study sedimentation rate.

PRL-299. Silt deposit

Wood from a brine well, depth $2.1 \mathrm{~m}$; sender's Sample RSK/49/4A. 


\section{PRL-300. Silt deposit}

$6160 \pm 110$

Wood from a brine well, depth 2.2m; sender's Sample RSK/41/4A.

PRL-301. Silt deposit

$8240 \pm 140$

Wood from a brine well, depth $4.1 \mathrm{~m}$; sender's Sample RSK/10/5A.

\section{PRL-302. Silt deposit}

$7560 \pm 140$

Wood from a brine well, depth $4.5 \mathrm{~m}$, sender's Sample RSK/24/4A.

\section{PRL-296. Naini Tal, India, talus deposit}

$540 \pm 100$

Wood from drill hole DHl, depth $61 \mathrm{~m}$ at Naini Tal $\left(29^{\circ} 23^{\prime} \mathrm{N}\right.$, $79^{\circ} 27^{\prime} \mathrm{E}$ ); subm by Dir Engg Geol Dn (East), Geol Survey India, Lucknow; sender's Sample 1. Comment: sample dated to study landslide history.

\section{Naliasar series, Rajasthan}

Naliasar, Jaipur Dist; subm by D P Agrawal, PRL, Ahmedabad. Comment: samples measured to study caliche formation.

\section{PRL-358. Caliche}

a) $12,750+280$

Caliche from lake basin, depth $0.05 \mathrm{~m}$; sender's Sample C-1.
b) $15,570+370$

a) $\mathbf{1 5 , 5 5 0}+\mathbf{5 5 0}$

b) $20,000+920$

PRL-359. Caliche

c) $22,320+640$

Caliche from lake basin, depth $0.1 \mathrm{~m}$; sender's Sample C-2.

\section{PRL-293. Nandipalli, India, fluvial deposit}

$23,670+690$

Shells from clayey silt deposit resting on Middle Paleolithic toolbearing gravel on Sagileru $\mathrm{R}$ at Nandipalli, Cuddapah Dist; subm by K Thimma Reddy.

\section{Navunda series, Karnataka}

Navunda village $\left(13^{\circ} 45^{\prime} \mathrm{N}, 74^{\circ} 38^{\prime} \mathrm{E}\right)$, South Canara Dist; subm by P S N Murty, Nat Min Dev Corp Ltd, Mangalore. Comment: samples measured to study lignite stratigraphy.

PRL-103. Lignite deposit

Lignite from a well $6 \mathrm{~m}$ deep and $1.5 \mathrm{~m}$ below water level. 


\section{PRL-132. Lignitized wood deposit}

Fragments of lignitized wood $9.4 \mathrm{~m}$ below surface and $1.2 \mathrm{~m}$ below water level. Comment: finite age probably due to contamination.

PRL-216. Odador, India, coastal aeolinite

$9390 \pm 140$

Limestone from semi-consolidated aeolinite $1.5 \mathrm{~km} \mathrm{SE}$ of Odador $\left(21^{\circ} 34^{\prime} \mathrm{N}, 69^{\circ} 40^{\prime} \mathrm{E}\right)$, Junagarh Dist, alt $+8 \mathrm{~m}$; subm by U B Mathur, Geol Survey India; sender's Sample MM 14. Comment: sample measured to date Late Quaternary coastal aeolinite.

\section{Pacific Ocean sediment series}

Box cores of calcareous sediments from Ontong Java Plateau $\left(4^{\circ}\right.$ $50^{\prime} \mathrm{N}$ to $0^{\circ} 07^{\prime} \mathrm{N}, 155^{\circ} 52^{\prime} \mathrm{E}$ to $163^{\circ} 42^{\prime} \mathrm{E}$ ), water depth 1597 to $4441 \mathrm{~m}$. Coll by W H Berger, subm by D Lal, PRL, Ahmedabad. Comment: samples measured to study sedimentation rate.

\begin{tabular}{|c|c|c|c|}
\hline Sample & Core no. & Core depth $(\mathrm{mm})$ & ${ }^{14} \mathrm{C}$ age \\
\hline PRL-360 & ERDC 88 BX 2 & 0 to 5 & $3060 \pm 150$ \\
\hline PRL-363 & $"$ & 50 to 60 & $5410 \pm 110$ \\
\hline PRL-362 & $"$ & 140 to 180 & $11,370 \pm 230$ \\
\hline PRL-361 & , & 230 to 260 & $17,460 \begin{array}{l}+440 \\
-420\end{array}$ \\
\hline PRL-364 & ERDC 102 BX 2 & $0-5$ & $7900 \pm 160$ \\
\hline PRL-366 & $"$ & $5-10$ & $7930 \pm 190$ \\
\hline PRL-367 & $"$ & $10-15$ & $4450 \pm 110$ \\
\hline PRL-368 & $"$ & $15-20$ & $8360 \pm 170$ \\
\hline PRL-369 & $"$ & $30-40$ & $5010 \pm 100$ \\
\hline PRL-370 & $"$ & $60-80$ & $6370 \pm 100$ \\
\hline PRL-371 & $"$ & $120-150$ & $10,570 \pm 180$ \\
\hline PRL-372 & , & $180-230$ & $\begin{array}{r}14,470+320 \\
-310\end{array}$ \\
\hline PRL-365 & , & $270-310$ & $22,050 \begin{array}{l}+560 \\
-520\end{array}$ \\
\hline PRL-284 & ERDC 123 BX 2 & $0-3$ & $2310 \pm 180$ \\
\hline PRL-285 & $"$ & $6.5-10.5$ & $2810 \pm 110$ \\
\hline PRL-286 & $"$ & $15.5-21$ & $3150 \pm 140$ \\
\hline PRL-326 & $"$ & $31-41$ & $3470 \pm 120$ \\
\hline PRL-327 & $"$ & $51-61$ & $3540 \pm 150$ \\
\hline PRL-346 & $"$ & $61-71$ & $3650 \pm 110$ \\
\hline PRL-328 & $"$ & $81-91$ & $4960 \pm 160$ \\
\hline PRL-329 & $"$ & 121-141 & $6550 \pm 120$ \\
\hline PRL-345 & $"$ & $181-201$ & $9110 \pm 130$ \\
\hline PRL-344 & $"$ & $261-291$ & $12,240 \pm 270$ \\
\hline PRL-332 & , & $321-361$ & 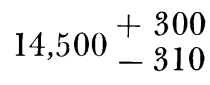 \\
\hline
\end{tabular}




\begin{tabular}{|c|c|c|c|}
\hline Sample & Core no. & Core depth $(\mathrm{mm})$ & ${ }^{14} \mathrm{C}$ age \\
\hline PRL-348 & ERDC 141 BX 2 & $0-5$ & $5790 \pm 170$ \\
\hline PRL-349 &, & $5-10$ & $6310 \pm 170$ \\
\hline PRL-350 & $"$ & $10-15$ & $6800 \pm 120$ \\
\hline PRL-351 & $"$ & $15-20$ & $6940 \pm 150$ \\
\hline PRL-352 & , & $30-40$ & $8560 \pm 140$ \\
\hline PRL-353 & $"$ & $60-80$ & 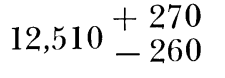 \\
\hline PRL-354 & ", & $140-180$ & $\begin{array}{r}28,730+1540 \\
-1290\end{array}$ \\
\hline PRL-356 & $"$ & $220-260$ & 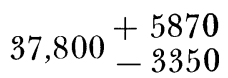 \\
\hline PRL-357 & , & $340-370$ & $>40,000$ \\
\hline
\end{tabular}

PRL-120. Pandiya Tivu, India, coastal sediment $2070 \pm 100$

Coral from Pandya Tivu $\left(78^{\circ} 13^{\prime}\right.$ E, $\left.8^{\circ} 45^{\prime} \mathrm{N}\right)$, alt $+3 \mathrm{~m}$; subm by A V N Sarma, Temple Univ, Philadelphia, Pennsylvania. Comment: sample measured to study sea-level changes on east coast of India.

PRL-145. Panetha, India, terrace deposit

$6470 \pm 180$

Pedocal with caliche from upper terrace on Narmada $R$ near Panetha, Broach Dist, depth 2.5m; subm by $\mathrm{N}$ Bedi, Geol Survey, India, Ahmedabad, sender's Sample GSI/NB/2.

PRL-30. Prabhas Patan, India, oyster shell bed

$20,825_{-540}^{+670}$

Shells from oyster bed on Hiran R, Junagarh Dist; subm by D P Agrawal. Comment: bed yielded Middle Paleolithic tools.

\section{Sankhu, Nepal}

Sankhu $\left(27^{\circ} 43^{\prime} \mathrm{N}, 88^{\circ} 28^{\prime} \mathrm{E}\right)$, Kathmandu Dist, subm by Vishnu Mittre, Birbal Sahni Inst Paleobot, Lucknow. Comment: samples were measured to date pollen sequence.

PRL-192. Carbonaceous clay deposit

$16,900+1010$

Peaty Clay II from exposed sec, depth 11.3m, sender's Sample Sankhu Boudh 1.

PRL-193. Carbonaceous clay deposit $>40,000$

Peaty Clay III from exposed sec, depth $13.75 \mathrm{~m}$, sender's Sample Sankhu Boudh 2.

PRL-194. Carbonaceous clay deposit $>40,000$

Peaty Clay III from exposed sec, depth $14.15 \mathrm{~m}$, sender's Sample Sankhu Boudh 3. 
PRL-195. Carbonaceous clay deposit

Peaty Clay III from exposed sec, depth $14.45 \mathrm{~m}$, sender's Sample Sankhu Boudh 4.

PRL-196. Carbonaceous clay deposit

Peaty Clay III from exposed sec, depth $14.75 \mathrm{~m}$, sender's Sample 5.

\section{Toshmaidan series, Jammu \& Kashmir}

Toshmaidan $\left(33^{\circ} 56^{\prime} \mathrm{N}, 73^{\circ} 31^{\prime} \mathrm{E}\right)$, Srinagar Dist; subm by $\mathrm{G}$ Singh, Australian Natl Univ, Canberra and D P Agrawal, PRL, Ahmedabad. Comment: samples were measured to date pollen sequence in a bog (Singh \& Agrawal, 1976, p 232).

\begin{tabular}{|c|c|c|c|c|c|c|}
\hline $\begin{array}{l}\text { Sample } \\
\text { no. }\end{array}$ & $\begin{array}{l}\text { Serial } \\
\text { no. }\end{array}$ & Depth (m) & Sample & $\begin{array}{l}\text { Sieve } \\
\text { fraction } \\
(\mathrm{m})\end{array}$ & $\begin{array}{c}\text { Pollen } \\
\text { stage }\end{array}$ & Date \\
\hline PRL-2B & II & .15 to .35 & Peat & 420 & $\mathrm{~g}$ & $2790 \pm 160$ \\
\hline PRL-3 & III & .5 to .7 & Peat & 420 & d & $9650 \pm 245$ \\
\hline PRL-4B & IV & .75 to .90 & Peat & 420 & d & $\begin{array}{r}+340 \\
10,005-380\end{array}$ \\
\hline PRL-5 & V & 1.25 to 1.4 & Peat & 420 & d & $\begin{array}{r}+585 \\
11,360-600\end{array}$ \\
\hline PRL-7 & VII & 2.05 to 2.2 & $\begin{array}{l}\text { Fine } \\
\text { organic mud }\end{array}$ & d & c & 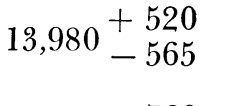 \\
\hline PRL-9 & IX & 2.8 to 2.95 & Clay mud & - & c & 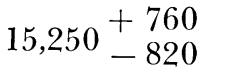 \\
\hline PRL-10 & $\mathrm{X}$ & 3.17 to 3.27 & Clay mud & 一 & $a-b$ & $14,760 \begin{array}{l}+1015 \\
-925\end{array}$ \\
\hline PRL-12 & XII & 3.37 to 3.5 & $\begin{array}{l}\text { Blue-gray } \\
\text { Lacustrine } \\
\text { clay }\end{array}$ & - & $a-b$ & $\begin{aligned} 13,830 & +900 \\
& -785\end{aligned}$ \\
\hline
\end{tabular}

\section{Tso-Kar series, Jammu \& Kashmir}

Tso-Kar $\left(33^{\circ} 20^{\prime} \mathrm{N}, 78^{\circ} \mathrm{E}\right)$ Ladakh Dist, subm by M Krishnamurthy, Geol Survey, India, Lucknow. Comment: samples measured to date paleoclimatic events.

PRL-259. Lacustrine deposit

$$
\mathbf{7 4 9 0} \pm \mathbf{1 9 0}
$$

Gastropod shells from lacustrine deposits at Tso-Kar, depth $2 \mathrm{~m}$.

PRL-261. Lacustrine deposit

$4840 \pm 170$

Carbon precipitate from lake sediment, depth $.6 \mathrm{~m}$.

\section{REFERENCES}

Agrawal, D P, Gupta, S K, and Kusumgar, Sheela, 1971, Tata Institute date list IX: Radiocarbon, v 13, p 442-449.

Stacul, G and Tusa, S, 1975, Report on the excavation at Aligrama (Swat, Pakistan) 1966, 1972: East and West, v 25, no. 3-4, p 291-321

Singh, Gurdip and Agrawal, D P, 1976, Radiocarbon evidence for deglaciation in northwestern Himalaya, India: Nature, v 260, no. 5548, p 232. 